\author{
R. Guarino, G. Domina \& S. Pignatti
}

\title{
Ellenberg's Indicator values for the Flora of Italy - first update: Pteridophyta, Gymnospermae and Monocotyledoneae
}

\begin{abstract}
Guarino, R., Domina, G. \& Pignatti, S.: Ellenberg's Indicator values for the Flora of Italy - first update: Pteridophyta, Gymnospermae and Monocotyledoneae. - F1. Medit. 22: 197-209. 2012. — ISSN: 1120-4052 printed, 2240-4538 online.

Ellenberg's indicator values are an useful tool to delineate the relationship between plants and environment, recognising to each species a functional role as biological indicator. In the frame of the second edition of the Pignatti's "Flora d'Italia", some new informative systems are under preparations, in order to support geobotanical/applied studies, including a complete and updated review of the Ellenberg's indicator values for the whole bulk of species mentioned in the flora of Italy. This first contribution includes a list of 380 species of Pteridophyta, Gymnospermae and Monocotiledoneae that complete the first assignment of the Ellenberg's indicator values to the flora of Italy, published in 2005. Besides, some methodological considerations on the attribution and the use of Ellenberg's indicator values are reported.
\end{abstract}

Key words: Eivs, bioindicator, plant ecology.

\section{Introduction}

Ellenberg's indicator values (Eivs) have been proposed to estimate the influence of main environmental factors in determining flora and vegetation changes on a considered surface area (Ellenberg 1974, 1996; Ellenberg \& al. 1992). Basing on a large observational and experimental evidence that ecological factors are the main determinant of structure and composition of plant communities, Ellenberg outlined the synecological preferences of all species belonging to the vascular flora of Central Europe, by means of numerical indices referring to 7 main environmental factors. These can be divided in two subgroups of three and four indices respectively: the first three indices refer to climatic variables: light conditions $(\mathrm{L})$, temperatures $(\mathrm{T})$, climatic continentality $(\mathrm{C})$; the last four deal with edaphic conditions: moisture $(\mathrm{U})$, reaction $(\mathrm{R})$, nutrient availability $(\mathrm{N})$, salinity $(\mathrm{S})$.

All Eivs are arranged in ordinal scales, in which only a nominal correlation is given with the physical/chemical parameters measuring the environmental variables to which they are referred. In the original scales (Ellenberg \& al. 1992), all indices were ranging between 1 and 9, with the only exceptions of $\mathrm{U}$, ranging between 1 and 12 and S, ranging between 0 and 9. In more recent times, S-range have been limited to 0-3 (Ellenberg 1996; Pignatti \& al. 2005). 
From Central Europe, Eivs have been extended eastwards to Poland by Zarzycky (1984) and to Hungary by Borhidi (1995). This application was not so problematic, owing to the large number of species in common and to the comparable latitudinal range. In subsequent years, they were proposed for the flora of the south-Aegean Region (Böhling \& al. 2002) and, for the first time, the opportunity to enlarge Ellenberg's scales in order to adapt them to warmer climatic conditions had to be discussed. Böhling \& al. (2002) deemed not necessary to modify the Ellenberg's ordinal scales, considering that they are adimensional and, therefore, can be re-calibrated at regional scale in order to make them coherent with the ecological behaviour of local plant species. In this way, for instance, Quercus pubescens happens to have $\mathrm{T}=6$ for the Cretan flora and $\mathrm{T}=8$ for the German one. The reason is rather obvious: downy oak in Germany indicates relatively warm and dry environmental conditions, while in Crete it is linked to relatively mesic habitats.

If Ellenberg's scales are left unchanged when moving to different latitudes, ecological comparisons with different regional floras become senseless. This problem became very evident when was tried to extend the Eivs to the Italian territory. Even if the ecological conditions in northern Italy are comparable to those of southern Germany and a significant number of species are in common, this is not true for the southern and insular Italian territories. For this reason an enlargement of the scales $\mathrm{L}$ and $\mathrm{T}$ to 12 values, in order to respect the statistic homogeneity with the original Ellenberg's assumption was proposed (Pignatti \& al. 2005).

The Eivs for the Italian flora published by Pignatti \& al. (2005) were based on the species list of the first edition of the renowned "Flora d'Italia" (Pignatti 1982), whose second edition is expected to be published (...hopefully!) by the end of the next year. Aim of this paper is to provide a first integration of the Eivs for the Italian Flora and, at the same time, to offer some methodological considerations on the attribution and the use of Ellenberg's indicator values.

\section{Material and methods}

The Eivs for the Italian flora published by Pignatti \& al. (2005) were including 5774 species of vascular plants. At the current state of the art, the second edition of "Flora d'Italia" will include 7292 species of vascular plants. This new species list incorporates and critically acknowledges hundreds of contributions to the knowledge of the Italian flora, among which the most relevant are the following: Aeschiman \& al. (2004); Arrigoni (2006-2010, 2011); Bacchetta \& al. (2009); Banfi \& Galasso (2011); Celesti-Grapow \& al. (2009, 2010); Conti \& al. (2005, 2007); Euro+Med (2006-); Giardina \& al. (2007); Peruzzi (2011); Raimondo \& al. (2010).

The difference from the species list of the first edition consists of 1518 species, of which 380 are pteridophytes, gymnosperms and monocots. These groups are the subject of our first contribution, where species are ordered according to APG (2006) and to Christenhustz \& al. (2011a, 2011b) for pteridophytes and gymnosperms.

In order to assign the new Eivs, all the criteria and recommendations adopted by Ellenberg \& al. (1992) have been followed, even if the criterion "per analogy" was leading our choices in most of the cases: given the growing stands and sociological behaviour of species $a$, the attribution of its Eivs followed in most of the cases the Eivs already assigned to the most frequent and abundant species in its growing stands or, in case of ambiguous magnitudes and correlations, the Eivs of species $a$ was arbitrarily assigned (basing on our experience) within the confidence interval delimited by the Eivs already assigned to the most frequent and abundant species in its growing stands. 


\section{Results}

Synecological requirements of a species may change along its distribution range, especially when moving to different latitudes. In order to extend the Eivs to the Italian flora, a data-base was implemented by Pignatti and his collaborators since more than twenty years. In such data-base, all the species of the first edition of "Flora d'Italia" were reported, as well as the phytosociological frame and results of ecological and ecophysiological measurements as far as available for each species. So far, that data-base was incremented with 380 newly assigned Eivs, which are reported in Annex 1. A second contribution, which will include the dicots still missing is currently under preparation.

On the website of the Herbarium Mediterraneum Panormitanum where Flora Mediterranea is published (http://www.herbmedit.org), the complete list of Eivs for the flora if Italy will be soon made available in the form of a Microsoft Excel ${ }^{\circledR}$ spreadsheet, as Electronic supplementary file in order to be easily imported in data-bases or readily used for data processing. This is an optimal condition for open-ended works, like floras and any kind of related data-base are per definition. The digital list of the Eivs will be periodically updated and adjusted, following the indications of all users, in order to progressively improve their accuracy. As already noted by Pignatti \& al. (2005) even the current list of Eivs has to be considered a first approximation, which may require a long period of adjustments and revisions. Ellenberg himself, when proposing the first edition of his indicator values, stated that it was a work in progress (Ellenberg 1974). The same was repeated in the following editions, defined as "further approximations".

While assigning the new Eivs, we reviewed, as well, the distribution frequency of all values in the list and we noticed that the value $\mathrm{L}=10$ was critically underrepresented in the list of Pignatti \& al. (2005). So, we reconsidered the pristine criterion of attributing $L=11$ to most of the Mediterranean species and assigned the value $\mathrm{L}=10$ to many synanthropic species and to many small sized annuals commonly found in Mediterranean garigues and perennial dry grasslands. This is just one example of how Eivs can change and may further change in the future, following new evidences.

Other examples of Eivs that have been modified from the edition of 2005 are the following:

- Arundo plinii: due to the re-evaluation of Arundo collina, the former species should be limited to the

loamy and clayish riverbeds of north-eastern Italy: Marche, Emilia-Romagna and Friuli-Venezia

Giulia. For this reason, its Eivs have been modified as follows: $C=6, U=6, R=5, N=5$

- Iris pseudopumila: $\mathrm{R}=6$, instead of $\mathrm{R}=4$

- Limodorum abortivum: $\mathrm{L}=4$, instead of $\mathrm{L}=\mathrm{X}$

\section{Discussion}

An important feature of Ellenberg's indices is that they do not express the physiological optimum of a given species, but to its synecological optimum, that is the optimal ecological requirements of a given species when interacting with other species. These interaction can vary from region to region and even from time to time. An approach to assess the significance of observed variations (in space and time) of Eivs has been proposed by Pignatti \& al. (2001a).

One might ask what is the meaning of Eivs in the era of environmental monitoring sensors and weatherproof digital loggers, which can be used for direct measurements of a large number of variables at any given time-interval. As already stated by Ellenberg himself, the indicator values do not replace measurements. Instead, they should be seen as practical tools to draft quick estimates for eco- 
logical interpretations of plant-species lists. On the other hand, they may help to design the distribution of a network of data loggers to corroborate and integrate with experimental evidence the indicator values. A bit like the interpolations of climate parameters: the higher is the number of meteorological stations recording real data to set and train models, the better is the accuracy and performance of predictive systems.

Until now, Eivs rely on quite a large number of application to "fingerprint" the ecological context of plant communities described by floristic compositions. For the Italian territory, the following papers contributed so far to corroborate the Eivs and their utiliy: Pignatti \& al. (1996, 2001a, 2001b); Pignatti (1998, 1999); Guarino \& Bernardini (2001); Fanelli \& al. (2007); Schwabe \& al. (2007); Gristina \& Marcenò (2008); Mossa \& al. (2008); Brunialti \& al. (2010); Pretto \& al. (2010); Pignatti E. \& Pignatti S. (in press); Guarino \& al. (in press).

Some authors (Durwen 1982; Böcker \& al. 1983; Kowarik \& Seidling 1989; Möller 1992), even if sometimes recognising a practical utility in calculating average Eivs of a species list, state that this is not mathematically correct, because Eivs are ordinal scales, without dimensional correlation with chemical/physical parameters. It is well demonstrated (see, for instance, Pignatti \& al. 2001a) that when the number samples (i.e. specie lists) is high enough, Eivs do fit the normal distribution, whose essential parameters are average and variance. So, in addition to non-parametric statistics, all kinds of statistical tests based on average and variance can be used. If only a small number of samples is available, a parametric statistic approach can be essayed anyway, because in the case of Ellenberg's scales it is always possible to transform data in their respective ranks, to better approximate the normal distribution and to create the conditions to use a parametric approach.

Moreover, several contributors demonstrated a fairly good correlation between Eivs and dimensional measurements of environmental variables (Degorski 1982; Seidling \& Rohner 1993; van der Maarel 1993; Möller 1997; Petersen 2000; Schaffers \& Sýkora 2000; Onori 2002). Thus, to perform parametric statistic tests and to use average indicator values referred to floras and plant communities should be considered always admissible, as already accepted by many authors. This greatly enlarges the utility of such indices.

\section{References}

Aeschimann, D., Lauber, K., Moser, D. M. \& Theurillat, J.-P. 2004: Flora Alpina. - Wien.

APG III, 2009. An update of the Angiosperm Phylogeny Group classification for the orders and families of flowering plants: APG III. - Bot. J. Linn. Soc. 161(2): 105-121. doi: 10.1111/j.10958339.2009.00996.x

Arrigoni, P. V. 2006-2010: La flora dell'Isola di Sardegna, 1-3. - Sassari.

— \& Viegi, L. 2011: La flora vascolare esotica spontaneizzata della Toscana. - Firenze.

Bacchetta, G., Mayoral Garcia Berlanga, O. \& Podda, L. 2009. Catálogo de la flora exótica de la isla de Cerdeña. - Fl. Montiberica 41: 35-61.

Banfi, E. \& Galasso, G. 2010. La Flora Esotica Lombarda. - Milano.

Böhling, N., Greuter, W. \& Raus, T. 2002: Zeigerwerte der Gefässpflanzen der Südägäis. - BraunBlanquetia 32: 3-107.

Böcker, R., Kowarik, I. \& Bornkamm, R. 1983. Untersuchungen zur Anwendung der Zeigerwerte nach Ellenberg. - Verh. Ges. Ökol. 11: 35-56.

Borhidi, A., 1995: Social behaviour types, the naturalness and relative ecological indicator values of the higher plants in the hungarian flora. - Acta Bot. Hungar. 39: 97-181. 
Brunialti, G., Frati, L., Aleffi, M., Marignani, M., Rosati, L., Burrascano, S. \& Ravera, S. 2010: Lichens and bryophytes as indicators of old growth features in Mediterranean forests. -Pl. Biosyst. 144(1): 221-233. doi: 10.1080/11263500903560959

Celesti-Grapow, L., Alessandrini, A., Arrigoni, P.V., Banfi, E., Bernardo, L., Bovio, M., Brundu, G., Cagiotti, M. R., Camarda, I., Carli, E., Conti, F., Fascetti, S., Galasso, G., Gubellini, L., La Valva, V., Lucchese, F., Marchiori, S., Mazzola, P., Peccenini, S., Poldini, L., Pretto, F., Prosser, F., Siniscalco, C., Villani, M.C., Viegi, L., Wilhalm, T. \& Blasi, C. 2009: Inventory of the non-native flora of Italy. - Pl. Biosyst. 143(2): 386-430. doi: $10.1080 / 11263500902722824$

—, - - - Assini, S., Banfi, E., Barni, E., Bovio, M., Brundu, G., Cagiotti, M. R., Camarda, I., Carli, E., Conti, F., Del Guacchio, E., Domina, G., Fascetti, S., Galasso, G., Gubellini, L., Lucchese, F., Medagli, P., Passalacqua, N. G., Peccenini, S., Poldini, L., Pretto, F., Prosser, F., Vidali, M., Viegi, L., Villani, M. C., Wilhalm, T. \& Blasi, C. 2010: Non-native flora of Italy: Species distribution and threats. - P1. Biosyst. 144(1): 12-28. doi: $10.1080 / 11263500903431870$

Christenhusz, M. J. M, Reveal, J. L., Farjon, A., Gardner, M. F., Mill, R. R. \& Chase, M. W. 2011a: A new classification and linear sequence of extant gymnosperms. - Phytotaxa 19: 55-70.

-, Zhang, X.-C. \& Schneider, H. 2011b. A linear sequence of extant families and genera of lycophytes and ferns. - Phytotaxa 19: 7-54.

Conti, F., Abbate, G., Alessandrini, A. \& Blasi, C. 2005: An annotated checklist of the Italian vascular flora. - Roma.

—, Alessandrini A., Bacchetta G., Banfi E., Barberis G., Bartolucci F., Bernardo L., Bonacquisti S., Bouvet D., Bovio M., Brusa G., Del Guacchio E., Foggi B., Frattini S., Galasso G., Gallo L., Gangale C., Gottschlich G., Grünanger P., Gubellini L., Iiriti G., Lucarini D., Marchetti D., Moraldo B., Peruzzi L., Poldini L., Prosser F., Raffaelli M., Santangelo A., Scassellati E., Scortegagna S., Selvi F., Soldano A., Tinti D., Ubaldi D., Uzunov D. \& Vidali M., 2007. Integrazioni alla checklist della flora vascolare italiana. - Nat. Vicentina 10(2006): 5-74.

Degorski, M. 1982: Usefullness if Ellenberg bioindicators in characteristic plan communities and forest habitats on the basis of data from the range Grabowy in Kampinos Forest. -Ekol. Pol. (Warsaw) 30: 453-477.

Durwen K. J., 1982: Zur Nutzung von Zeigerwerten und artspezifischen Merkmalen der Gefässpflanzen Mitteleuropas für Zwecke der Landschaftökologie und -planung mit Hilfe der EDV. - Arbeitsber. Lehrst. Landschaftökol. Münster 5: 1-138.

Ellenberg, H. 1974: Zeigerwerte der Gefässpflanzen Mitteleuropas. - Scripta Geobot. 9: 1-97. - 1996. Vegetation Mitteleuropas mit den Alpen ( $5^{\text {th }}$ edition). - Stuttgart.

—, Weber, H. E., Düll, R., Wirth, V., Werner, W. \& Paulißen, D. 1992: Zeigerwerte von Pflanzen in Mitteleuropa. - Scripta Geobot. 18(2): 1-258.

Euro+Med, 2006-: Euro+Med PlantBase - the information resource for Euro-Mediterranean plant diversity. -URL: http://ww2.bgbm.org/EuroPlusMed/ [accessed 2012-10-16].

Fanelli, G., Lestini, M. \& Serafini Sauli, A. 2007: Floristic gradients of herbaceous vegetation and $\mathrm{P} / \mathrm{N}$ ratio in soil in a Mediterranean area. - Pl. Ecol. doi 10.1007/s11258-007-9287-8

Giardina, G., Raimondo, F. M. \& Spadaro, V. 2007: A catalogue of plants growing in Sicily. Bocconea 20: 5-582.

Gristina, A. S. \& Marcenò, C. 2008. Gli indici di bioindicazione di Pignatti-Ellenberg nello studio floristico-vegetazionale del promontorio di Capo Zafferano (Sicilia nord-occidentale). Naturalista Sicil., s. 4, 32(1-2): 61-96. 
Guarino, R. \& Bernardini, A. 2002: Indagine sulla diversità floro-vegetazionale del comprensorio del cuoio (Toscana centro-settentrionale). - San Miniato (PI).

-, Bazan, G. \& Paura, B. in press: Downy-oak woods of Italy: phytogeographical remarks on a controversial taxonomic and ecologic issue. - Braun-Blanquetia.

Kowarik, I. \& Seidling, W. 1989: Zeigerwertberechnungen nach Ellenberg, zur Problemen und Einschränkungen einer sinnvollen Methode. - Lanschaft. Stadt 21(4): 132-143.

Möller, H. 1992: Zur Verwendung des Medians bei Zeigerwertberechnungen nach Ellenberg. Tuexenia 12: 25-28.

— 1997: Reaktions- und Stickstoffzahlen nach Ellenberg als Indikatoren für die Humusform in terrestrischen Waldökosystemen im Raum Hannover. - Tuexenia 17: 349-365.

Mossa, L., Aru, A., Fogu, M. C., Guarino, R. \& Zavattero, L. 2008: Studio geobotanico del Parco Eolico di Ulassai. - Catania.

Onori L. (ed.) 2002: Un nuovo approccio per la valutazione della biodiversità. - ANPA; Manuali e Linee Guida 14: 1-103.

Peruzzi, L. 2010. Checklist dei generi e delle famiglie della flora vascolare italiana. - Inform. Bot. Ital. 42(1): 151-170.

Petersen, J. 2000. Die dünentalvegetation der Wattenmeer-Inseln in der südlichen Nordsee. Husum.

Pignatti, E. \& Pignatti, S. in press: Plant life in the Dolomites. - Naturmuseum Bozen.

Pignatti, S. 1982: Flora d'Italia, 1-3. - Bologna.

- 1998. I Boschi d'Italia. - Torino.

-1999. La phytosociologie Braun-Blanquetiste et ses perspectives. - Coll. Phytosoc. 27: 1-15.

-, Bianco, P. M., Fanelli, G., Guarino, R., Petersen, L. \& Tescarollo, P. 2001: Reliability and effectiveness of Ellenberg's indices in checking flora and vegetation changes induced by climatic variations. - Pp 281-304 in: Walter, G.-R., Burga, C. A. \& Edwards, P. J. (eds.), Fingerprints of Climate Changes: adapted behaviour and shifting species ranges - New York \& London.

—, Bianco, P. M., Tescarollo, P. \& Scarascia-Mugnozza, G. T. 2001b: La vegetazione della Tenuta Presidenziale di Castelporziano. In: Il sistema ambientale della Tenuta Presidenziale di Castelporziano. - Accad. Sci. Scritti Doc. 26(2): 441-710.

—, Ellenberg H. \& Pietrosanti S. 1996: Ecograms for phytosociological tables based on Ellenberg's Zeigerwerte. - Ann. Bot. 54: 5-14.

-, Menegoni, P. \& Pietrosanti, S. 2005: Biondicazione attraverso le piante vascolari. Valori di indicazione secondo Ellenberg (Zeigerwerte) per le specie della Flora d'Italia. - Braun-Blanquetia 39: 1-97.

Pretto, L., Celesti-Grapow, L., Carli, L. \& Blasi, C. 2010: Influence of past land use and current human disturbance on non-native plant species on small Italian islands. - Pl. Ecol. doi: 10.1007/s11258-010-9751-8.

Raimondo, F. M., Domina, G. \& Spadaro, V. 2010: Checklist of the vascular flora of Sicily. - Quad. Bot. Amb. Appl. 21(2010): 181-252.

Schwabe, A., Kratochwil, A. \& Pignatti, S. 2007: Plant indicator values of a high-phytodiversity country (Italy) and their evidence, exemplified for model areas with climatic gradients in the southern inner Alps. - Flora 202: 339-349.

Seidling, W. \& Rohner, M. S. 1993: Zusammenhänge zwischen Reaktions-Zeigerwerten und bodenchemischen Parametern am Beispiel von Waldbodenvegetation. - Phytocoenologia 23: 301-317.

Schaffers, A. P. \& Sýkora, K. V. 2000: Reliability of Ellenberg indicator values for moisture, nitrogen and soil reaction: a comparison with field measurements. - J. Veg. Sci. 11: 225-244. 
van der Maarel, E. 1993: Relations between sociological-ecological species groups and Ellenberg indicator values. - Phytocoenologia 23: 343-362.

Zarzycky, K. 1984: Indicator values of vascular plants in Poland. - Krakow Inst. Bot. Polska Akad. Nauk.

Addresses of the authors:

Riccardo Guarino ${ }^{1}$, Gianniantonio Domina ${ }^{1} \&$ Sandro Pignatti ${ }^{2}$

${ }^{1}$ University of Palermo, Dept. of Environmental Biology and Biodiversity, via Archirafi, 38 - 90123 Palermo (Italy); E-mail: riccardo.guarino@unipa.it

${ }^{2}$ University of Rome“La Sapienza", Dept. of Environmental Biology, piazza A. Moro, 5 - 00165 Rome (Italy) 
Annex 1. Eivs assigned to the considered species. The value "X" stands for "undetermined" i.e. species with a very wide ecological amplitude for the considered variable. The value " 0 " stands for "unknown" (i.e. data deficient), with the exception of S, where "0" means "no salinity". Additional informations on the meaning of Eivs can be found in Pignatti \& al. (2005).

\begin{tabular}{|c|c|c|c|c|c|c|c|c|c|}
\hline Code & Family & Taxon & L & $T$ & C & $\mathrm{U}$ & $\mathbf{R}$ & $\mathbf{N}$ & $\mathbf{S}$ \\
\hline 9903006 & Lycopodiaceae & Diphasiastrum oellgaardii Stoor, Boudrie, Jérôme, Horn \& Bennert & 8 & 2 & 4 & 5 & 2 & 2 & 0 \\
\hline 9905015 & Isoetaceae & Isoetes todaroana Troia \& Raimondo & 7 & 9 & 4 & 10 & 1 & 1 & 0 \\
\hline 9945001 & Hymenophyllaceae & Vandenboschia speciosa (Willd.) G. Kunkel & 7 & 10 & 2 & 3 & 6 & 4 & 0 \\
\hline 9923022 & Aspleniaceae & Asplenium balearicum Shivas & 7 & 10 & 2 & 2 & 6 & 1 & 0 \\
\hline 9929005 & Athyriaceae & Cystopteris alpina (Lam.) Desv. & 3 & 3 & 4 & 5 & 3 & 3 & 0 \\
\hline 9929006 & Athyriaceae & Cystopteris diaphana (Bory) Blasdell & 5 & 4 & 4 & 5 & 1 & 1 & 0 \\
\hline 9944002 & Dryopteridaceae & Cyrtomium falcatum (L. fil.) C. Presl & 3 & 4 & 4 & 5 & 4 & 5 & 0 \\
\hline 9934013 & Dryopteridaceae & Dryopteris pallida (Bory) Maire \& Petitm. subsp. pallida & 6 & 7 & 5 & 4 & 6 & 5 & 0 \\
\hline 9934014 & Dryopteridaceae & Dryopteris submontana (Fraser-Jenk. \& Jermy) Fraser-Jenk. & 6 & 6 & 5 & 5 & 6 & 3 & 0 \\
\hline 9934015 & Dryopteridaceae & Dryopteris remota (A. Braun ex Döll) Druce & 6 & 6 & 5 & 5 & 5 & 3 & 0 \\
\hline 9934016 & Dryopteridaceae & Dryopteris expansa (C. Presl) Fraser-Jenk. \& Jermy & 6 & 6 & 4 & 4 & 5 & 3 & 0 \\
\hline 9946001 & Nephrolepidaceae & Nephrolepis cordifolia C. Presl & 6 & 6 & 4 & 4 & 2 & 5 & 0 \\
\hline 0027001 & Pinaceae & Pseudotsuga menziesii (Mirbel) Franco & 5 & 4 & 6 & 1 & 5 & 0 & 0 \\
\hline 0023002 & Pinaceae & Cedrus deodara (D. Don) G. Don & 7 & 7 & 4 & 2 & 7 & 2 & 0 \\
\hline 0023003 & Pinaceae & Cedrus libani A. Richard & 7 & 7 & 4 & 2 & 7 & 2 & 0 \\
\hline 0023001 & Pinaceae & Cedrus atlantica (Endl.) Carrière & 11 & 8 & 3 & 2 & $\mathrm{X}$ & 2 & 0 \\
\hline 0022014 & Pinaceae & Pinus canariensis Sweet & 11 & 10 & 2 & 2 & $\mathrm{X}$ & 2 & 0 \\
\hline 0022018 & Pinaceae & Pinus wallichiana Jackson & 8 & 3 & 5 & 0 & $\mathrm{X}$ & 3 & 0 \\
\hline 0022020 & Pinaceae & Pinus strobus L. & 7 & 7 & 5 & 3 & $\mathrm{X}$ & 3 & 0 \\
\hline 0022003 & Pinaceae & Pinus radiata Don & 7 & 7 & 4 & 3 & $\mathrm{X}$ & 3 & 0 \\
\hline 0032001 & Taxodiaceae & Sequoia sempervirens (Lamb.) Endl. & 11 & 8 & 4 & 2 & 4 & 2 & 0 \\
\hline 0035001 & Taxodiaceae & Taxodium distichum (L.) Richard & 7 & 7 & 6 & 3 & 5 & 3 & 0 \\
\hline 0034001 & Taxodiaceae & Cryptomeria japonica (L. fil.) Don & 7 & 7 & 6 & 3 & 5 & 3 & 0 \\
\hline 0043002 & Cupressaceae & Cupressus macrocarpa Hartw. & 7 & 7 & 6 & 3 & $\mathrm{X}$ & 3 & 0 \\
\hline 0043004 & Cupressaceae & Cupressus arizonica Green & 7 & 7 & 6 & 3 & 5 & 3 & 0 \\
\hline 0044001 & Cupressaceae & Chamaecyparis lawsoniana (Murray) Parl. & 7 & 7 & 4 & 3 & 5 & 3 & 0 \\
\hline 0042001 & Cupressaceae & Thuja occidentalis L. & 7 & 7 & 6 & 3 & 6 & 3 & 0 \\
\hline 0042002 & Cupressaceae & Thuja orientalis L. & 7 & 7 & 6 & 3 & 6 & 3 & 0 \\
\hline 0045010 & Cupressaceae & Juniperus virginiana $\mathrm{L}$. & 7 & 7 & 4 & 3 & 5 & 3 & 0 \\
\hline 0010001 & Ginkgoaceae & Ginkgo biloba L. & 7 & 6 & 7 & 2 & 6 & 2 & 0 \\
\hline 0046005 & Ephedraceae & Ephedra foeminea Forssk. & 11 & 8 & 4 & 3 & $\mathrm{X}$ & 2 & 0 \\
\hline 0046008 & Ephedraceae & Ephedra podostylax Boiss. & 11 & 10 & 4 & 3 & $\mathrm{X}$ & 2 & 0 \\
\hline 0046006 & Ephedraceae & Ephedra dubia Boiss. & 11 & 10 & 5 & 3 & $\mathrm{X}$ & 2 & 0 \\
\hline 0046007 & Ephedraceae & Ephedra negrii Nouviant & 11 & 8 & 5 & 3 & $\mathrm{X}$ & 2 & 0 \\
\hline 2513005 & Nymphaeaceae & Nymphaea mexicana Zucc. & 8 & $\mathrm{X}$ & 4 & 12 & 7 & 7 & 0 \\
\hline 2654001 & Magnoliaceae & Liriodendron tulipifera L. & 8 & 7 & 3 & 3 & 7 & 5 & 0 \\
\hline 2651001 & Magnoliaceae & Magnolia grandiflora L. & 8 & 7 & 4 & 3 & 7 & 5 & 0 \\
\hline 2174012 & Aristolochiaceae & Aristolochia clusii Lojac. & 7 & 8 & 3 & 3 & 6 & 3 & 0 \\
\hline 2174013 & Aristolochiaceae & Aristolochia navicularis E. Nardi & 8 & 10 & 4 & 3 & 5 & 2 & 0 \\
\hline 0777009 & Araceae & Arum apulum (Carano) P. C. Boyce & 5 & 8 & 4 & 4 & 5 & 5 & 0 \\
\hline 0791001 & Araceae & Pistia stratiotes L. & 7 & 7 & 4 & 12 & 7 & 8 & 0 \\
\hline 0795005 & Araceae & Lemna minuta Kunth & 7 & 6 & 5 & 12 & 7 & 8 & 0 \\
\hline 0795006 & Araceae & Lemna valdiviana Phil. & 7 & 6 & 5 & 12 & 7 & 8 & 0 \\
\hline 0085001 & Hydrocharitaceae & Halophila stipulacea (Forssk.) Asch. & 5 & 3 & 1 & 12 & 8 & 7 & 8 \\
\hline 0062005 & Cymodoceaceae & Zannichellia obtusifolia Talavera, García Murillo \& Smit & 7 & 0 & 2 & 12 & 7 & 6 & 1 \\
\hline 0062006 & Cymodoceaceae & Zannichellia peltata Bertol. & 6 & 0 & 2 & 12 & 7 & 6 & 1 \\
\hline 0062002 & Cymodoceaceae & Zannichellia major Boenning. \& Rchb. & 6 & 0 & 2 & 12 & 7 & 6 & 1 \\
\hline 0062003 & Cymodoceaceae & Zannichellia pedunculata Rchb. & 6 & 0 & 2 & 12 & 7 & 6 & 1 \\
\hline 0062004 & Cymodoceaceae & Zannichellia melitensis Brullo, Giusso \& Lanfranco & 7 & 0 & 2 & 12 & 7 & 6 & 1 \\
\hline 0960003 & Melanthiaceae & Veratrum Iobelianum Bernh. & 8 & 4 & 5 & 7 & 4 & 5 & 0 \\
\hline 0978027 & Colchicaceae & Colchicum triphyllum Kunze & 6 & 6 & 3 & 3 & 6 & 6 & 0 \\
\hline 0978028 & Colchicaceae & Colchicum actupii Fridlender & 6 & 7 & 4 & 3 & 6 & 7 & 0 \\
\hline 0978011 & Colchicaceae & Colchicum gonarei Camarda & 8 & 7 & 3 & 3 & 6 & 4 & 0 \\
\hline 1048032 & Liliaceae & Gagea tisoniana Peruzzi, Bartolucci, Frignani \& Minutillo & 9 & 4 & 3 & 3 & 5 & 3 & 0 \\
\hline 1048033 & Liliaceae & Gagea polidorii J.-M. Tison & 8 & 5 & 5 & 3 & 4 & 3 & 0 \\
\hline 1048034 & Liliaceae & Gagea dubia A. Terracc. & 9 & 4 & 3 & 3 & 4 & 3 & 0 \\
\hline 1048035 & Liliaceae & Gagea luberonensis J.-M. Tison & 8 & 7 & 3 & 3 & 4 & 3 & 0 \\
\hline 1048036 & Liliaceae & Gagea peduncularis (J. \& C. Presl) Pascher & 8 & 11 & 3 & 3 & 5 & 3 & 0 \\
\hline 1048037 & Liliaceae & Gagea sicula Lojac. & 9 & 4 & 3 & 3 & 5 & 3 & 0 \\
\hline 1048024 & Liliaceae & Gagea lojaconoi Peruzzi & 9 & 4 & 3 & 3 & 5 & 3 & 0 \\
\hline
\end{tabular}


Annex 1. continued.

\begin{tabular}{|c|c|c|c|c|c|c|c|c|c|}
\hline 1074025 & Liliaceae & $\begin{array}{l}\text { Fritillaria persica L. } \\
\end{array}$ & 8 & 6 & 5 & 3 & 6 & 3 & 0 \\
\hline 1074026 & Liliaceae & Fritillaria imperialis $\mathrm{L}$. & 8 & 6 & 5 & 3 & 6 & 3 & 0 \\
\hline 1072007 & Liliaceae & Lilium chalcedonicum L. & 6 & 4 & 5 & 4 & 6 & 5 & 0 \\
\hline 1113017 & Asparagaceae & Asparagus sprengeri Regel & 8 & 10 & 3 & 2 & 4 & 2 & 0 \\
\hline 1113001 & Asparagaceae & Elide asparagoides (L.) Kerguélen & 5 & 7 & 5 & 5 & 8 & 6 & 0 \\
\hline 1219002 & Agavaceae & Agave salmiana Otto ex Salm-Dyck & 11 & 10 & 2 & 2 & $\bar{X}$ & 2 & 0 \\
\hline 1219003 & Agavaceae & Agave sisalana Perrine & 11 & 10 & 2 & 2 & $\bar{X}$ & 2 & 0 \\
\hline 1219004 & Agavaceae & Agave vivipara $\mathrm{L}$. & 11 & 10 & 2 & 2 & $x$ & 2 & 0 \\
\hline 1103003 & Agavaceae & Yucca aloifolia L. & 9 & 10 & 2 & 2 & 6 & 2 & 0 \\
\hline 1103001 & Agavaceae & Yucca filamentosa $\mathrm{L}$. & 9 & 10 & 2 & 2 & 6 & 2 & 0 \\
\hline 1103002 & Agavaceae & Yucca gloriosa L. & 9 & 10 & 2 & 2 & 6 & 2 & 0 \\
\hline 1086002 & Hyacinthaceae & Scilla luciliae (Boiss.) Speta & 5 & 6 & 5 & 6 & 7 & 6 & 0 \\
\hline 1086004 & Hyacinthaceae & Scilla amoena L. & 5 & 6 & 5 & 6 & 7 & 6 & 0 \\
\hline 1086010 & Hyacinthaceae & Chouardia litardierei (Breistr.) Speta & 7 & 7 & 5 & 3 & 5 & 5 & 0 \\
\hline 1086027 & Hyacinthaceae & Prospero elisae Speta & 8 & 8 & 5 & 2 & 6 & 3 & 0 \\
\hline 1086026 & Hyacinthaceae & Prospero corsica (Boullu) J. M. Tison & 8 & 8 & 4 & 2 & 6 & 3 & 0 \\
\hline 1086023 & Hyacinthaceae & Prospero hierae C. Brullo, Brullo, Giusso, Pavone \& Salmeri & 8 & 9 & 4 & 2 & 6 & 3 & 0 \\
\hline 1086024 & Hyacinthaceae & Oncostema sicula (Tineo ex Guss.) Speta & 8 & 7 & 3 & 2 & 6 & 3 & 0 \\
\hline 1086025 & Hyacinthaceae & Oncostema dimartinoi (Brullo \& Pavone) F. Conti \& Soldano & 10 & 9 & 3 & 1 & 6 & 3 & 0 \\
\hline 1093803 & Hyacinthaceae & Brimeura amethystina (L.) Salisb. & 7 & 7 & 5 & 3 & 5 & 5 & 0 \\
\hline 1093909 & Hyacinthaceae & Bellevalia pelagica C. Brullo, Brullo \& Pasta & 10 & 10 & 3 & 2 & 6 & 3 & 0 \\
\hline 1095010 & Hyacinthaceae & Muscari armeniacum Leichtlin ex Baker & 7 & 7 & 6 & 4 & 6 & 3 & 0 \\
\hline 1095801 & Hyacinthaceae & Muscarimia macrocarpa (Sweet) Garbari & 7 & 7 & 5 & 4 & 6 & 3 & 0 \\
\hline 1095802 & Hyacinthaceae & Muscarimia muscari (L.) Losinsk. & 7 & 7 & 6 & 4 & 6 & 3 & 0 \\
\hline 1089043 & Hyacinthaceae & Stellarioides canaliculata Medik. & 8 & 11 & 5 & 2 & 5 & 3 & 0 \\
\hline 1049003 & Alliaceae & Allium senescens subsp. montanum (Fries) Holub & 8 & 7 & 5 & 3 & 6 & 4 & 0 \\
\hline 1049021 & Alliaceae & Allium fistulosum $\mathrm{L}$. & 8 & 8 & 5 & 6 & 5 & 5 & 0 \\
\hline 1049033 & Alliaceae & Allium moly L. & 8 & 8 & 5 & 6 & 5 & 5 & 0 \\
\hline 1049144 & Alliaceae & Allium permixtum Guss. & 7 & 7 & 5 & 3 & 6 & 4 & 0 \\
\hline 1049114 & Alliaceae & Allium vernale Tineo & 10 & 9 & 4 & 2 & 4 & 1 & 0 \\
\hline 1049137 & Alliaceae & Allium franciniae Brullo \& Pavone & 11 & 9 & 4 & 3 & 7 & 1 & 0 \\
\hline 1049153 & Alliaceae & Allium samniticum Brullo, Pavone \& Salmeri & 8 & 8 & 4 & 3 & 6 & 5 & 0 \\
\hline 1049151 & Alliaceae & Allium panormitanum Brullo, Pavone \& Salmeri & 8 & 8 & 4 & 3 & 6 & 5 & 0 \\
\hline 1049152 & Alliaceae & Allium pelagicum Brullo, Pavone \& Salmeri & 11 & 10 & 4 & 3 & 7 & 1 & 0 \\
\hline 1049143 & Alliaceae & Allium pentadactyli Brullo, Pavone \& Spamp. & 8 & 8 & 4 & 3 & 6 & 5 & 0 \\
\hline 1049161 & Alliaceae & Allium lojaconoi Brullo, Lanfranco \& Pavone & 11 & 9 & 4 & 3 & 7 & 1 & 0 \\
\hline 1049141 & Alliaceae & Allium lopadusanum Bartolo, Brullo \& Pavone & 11 & 10 & 4 & 3 & 7 & 1 & 0 \\
\hline 1049156 & Alliaceae & Allium garganicum Brullo, Pavone Salmeri \& Terrasi & 8 & 8 & 4 & 3 & 6 & 5 & 0 \\
\hline 1049133 & Alliaceae & Allium calabrum (N. Terracc.) Brullo, Pavone \& Salmeri & 8 & 8 & 4 & 3 & 6 & 5 & 0 \\
\hline 1049131 & Alliaceae & Allium apulum Brullo, Guglielmo, Pavone \& Salmeri & 8 & 8 & 4 & 3 & 6 & 5 & 0 \\
\hline 1049135 & Alliaceae & Allium dentiferum Webb \& Berthel. & 11 & 8 & 4 & 3 & 6 & 3 & 0 \\
\hline 1049149 & Alliaceae & Allium julianum Brullo, Gangale \& Uzunov & 7 & 7 & 4 & 3 & 6 & 4 & 0 \\
\hline 1049136 & Alliaceae & Allium diomedeum Brullo, Guglielmo, Pavone \& Salmeri & 11 & 9 & 4 & 3 & 7 & 1 & 0 \\
\hline 1049140 & Alliaceae & Allium lehmannii Lojac. & 9 & 10 & 4 & 2 & 4 & 2 & 0 \\
\hline 1049129 & Alliaceae & Allium agrigentinum Brullo \& Pavone & 9 & 10 & 4 & 2 & 4 & 2 & 0 \\
\hline 1049134 & Alliaceae & Allium castellanense (Garbari, Miceli \& Raimondo) Brullo, Guglielmo, Pavone \& Salmeri & 9 & 10 & 4 & 2 & 4 & 2 & 0 \\
\hline 1049132 & Alliaceae & Allium garbarii Peruzzi & 9 & 10 & 4 & 2 & 4 & 2 & 0 \\
\hline 1049147 & Alliaceae & Allium savii Parl. & 8 & 7 & 5 & 3 & 5 & 6 & 0 \\
\hline 1049130 & Alliaceae & Allium anzalonei Brullo, Pavone \& Salmeri & 7 & 7 & 6 & 3 & 6 & 5 & 0 \\
\hline 1049075 & Alliaceae & Allium sativum $\mathrm{L}$. & 8 & 8 & 5 & 6 & 5 & 5 & 0 \\
\hline 1049146 & Alliaceae & Allium porrum $\mathrm{L}$. & 8 & 8 & 5 & 6 & 5 & 5 & 0 \\
\hline 1049138 & Alliaceae & Allium hemisphaericum (Sommier) Brullo & 8 & 9 & 5 & 2 & 6 & 2 & 0 \\
\hline 1049157 & Alliaceae & Nothoscordum inodorum (Aiton) Nicholson & 10 & 9 & 4 & 2 & 4 & 1 & 0 \\
\hline 1061001 & Alliaceae & Ipheion uniflorum (Graham) Raf. & 11 & 8 & 3 & 2 & 4 & 2 & 0 \\
\hline 1176001 & Amaryllidaceae & Amaryllis bella-donna L. & 8 & 10 & 4 & 2 & 4 & 2 & 0 \\
\hline 1185003 & Amaryllidaceae & Sternbergia sicula Tineo ex Guss. & 7 & 6 & 3 & 4 & 5 & 4 & 0 \\
\hline 1174009 & Amaryllidaceae & Leucojum nicaeense Ardoino & 9 & 9 & 3 & 1 & 5 & 2 & 0 \\
\hline 1172005 & Amaryllidaceae & Galanthus reginae-olgae Orph. & 5 & 7 & 4 & 5 & 7 & 7 & 0 \\
\hline 1172003 & Amaryllidaceae & Galanthus elwesii Hooker & 5 & 7 & 4 & 5 & 7 & 7 & 0 \\
\hline 1201036 & Amaryllidaceae & Narcissus etruscus Parl. & 8 & 8 & 4 & 4 & 5 & 4 & 0 \\
\hline 1201004 & Amaryllidaceae & Narcissus papyraceus Ker Gawl. & 8 & 8 & 4 & 4 & 5 & 4 & 0 \\
\hline 1201031 & Amaryllidaceae & Narcissus incomparabilis Mill. & 8 & 8 & 4 & 4 & 5 & 4 & 0 \\
\hline 1201033 & Amaryllidaceae & Narcissus medioluteus Mill. & 8 & 8 & 4 & 4 & 5 & 4 & 0 \\
\hline 1202003 & Amaryllidaceae & Pancratium linosae Soldano \& F. Conti & 11 & 10 & 3 & 1 & 6 & 1 & 0 \\
\hline
\end{tabular}


Annex 1. continued.

\begin{tabular}{|c|c|c|c|c|c|c|c|c|c|}
\hline 0980008 & Xanthorrhoeaceae & Asphodelus ayardii Jahand. \& Maire & 9 & 8 & 3 & 2 & 3 & 5 & 0 \\
\hline 1026004 & Xanthorrhoeaceae & Aloe vera (L.) Burm. fil. & 9 & 10 & 2 & 2 & 6 & 2 & 0 \\
\hline 1026007 & Xanthorrhoeaceae & Aloe succotrina All. & 9 & 10 & 2 & 2 & $\mathrm{X}$ & 2 & 0 \\
\hline 1259046 & Iridaceae & Crocus siculus Tineo & 8 & 8 & 6 & 4 & 6 & 4 & 0 \\
\hline 1259047 & Iridaceae & Crocus ilvensis Peruzzi \& Carta & 9 & 10 & 3 & 3 & 3 & 1 & 0 \\
\hline 1259045 & Iridaceae & Crocus sativus $\mathrm{L}$. & 6 & 8 & 3 & 3 & 6 & 2 & 0 \\
\hline 1261013 & Iridaceae & Romulea bocchierii Frignani \& liriti & 8 & 9 & 4 & 7 & 4 & 3 & 0 \\
\hline 1261015 & Iridaceae & Romulea rosea (L.) Eckl. & 8 & 9 & 4 & 3 & 4 & 3 & 0 \\
\hline 1316001 & Iridaceae & Freesia refracta (Jacq.) Eckl. ex Klatt & 7 & 9 & 4 & 3 & 6 & 4 & 0 \\
\hline 1306001 & Iridaceae & Tritonia crocosmiiflora (Lemoine) Nicholson & 7 & 9 & 4 & 4 & 6 & 4 & 0 \\
\hline 1311009 & Iridaceae & Gladiolus vexillaris Martelli & 9 & 9 & 5 & 3 & 5 & 3 & 0 \\
\hline 1312001 & Iridaceae & Chasmanthe bicolor (Gasp.) N. E. Br. & 8 & 10 & 4 & 2 & 6 & 2 & 0 \\
\hline 1264046 & Iridaceae & Iris unguicularis Poir. & 8 & 10 & 5 & 2 & 6 & 2 & 0 \\
\hline 1264047 & Iridaceae & Iris lactea Pallas & 8 & 8 & 6 & 3 & 5 & 4 & 0 \\
\hline 1264048 & Iridaceae & Iris orientalis $\mathrm{L}$. & 8 & 8 & 6 & 3 & 5 & 4 & 0 \\
\hline 1264049 & Iridaceae & Iris japonica Thunb. & 8 & 8 & 6 & 3 & 5 & 4 & 0 \\
\hline 1264050 & Iridaceae & Iris tectorum Maxim. & 8 & 8 & 6 & 3 & 5 & 4 & 0 \\
\hline 1264051 & Iridaceae & Iris perrieri Simonet ex Fournier & 7 & 7 & 6 & 2 & 4 & 1 & 0 \\
\hline 1264041 & Iridaceae & Iris pallida Lam. & 7 & 7 & 5 & 3 & 4 & 3 & 0 \\
\hline 1264044 & Iridaceae & Iris sambucina L. & 7 & 7 & 5 & 3 & 4 & 3 & 0 \\
\hline 1264052 & Iridaceae & Iris squalens L. & 7 & 7 & 5 & 3 & 4 & 3 & 0 \\
\hline 1264045 & Iridaceae & Iris setina Colas. & 8 & 9 & 5 & 2 & 6 & 2 & 0 \\
\hline 1264037 & Iridaceae & Iris bicapitata Colas. & 8 & 9 & 4 & 2 & 6 & 2 & 0 \\
\hline 1264053 & Iridaceae & Iris statellae Tod. & 8 & 10 & 4 & 2 & 6 & 2 & 0 \\
\hline 1264031 & Iridaceae & Iris benacensis Kerner & 7 & 7 & 5 & 3 & 4 & 3 & 0 \\
\hline 1264042 & Iridaceae & Iris relicta Colas. & 7 & 7 & 5 & 3 & 5 & 4 & 0 \\
\hline 1264043 & Iridaceae & Iris sabina N. Terracc. & 8 & 10 & 4 & 2 & 6 & 2 & 0 \\
\hline 1264036 & Iridaceae & Iris albicans Lange & 7 & 7 & 5 & 3 & 4 & 3 & 0 \\
\hline 1404006 & Orchidaceae & Nigritella corneliana (Beauverd) Gölz \& H. R. Reinhard & 8 & 3 & 4 & 4 & 7 & 3 & 0 \\
\hline 1404007 & Orchidaceae & Nigritella widderi Teppner \& E. Klein & 8 & 3 & 4 & 4 & 7 & 3 & 0 \\
\hline 1404008 & Orchidaceae & Nigritella buschmanniae Teppner \& Ster & 8 & 3 & 4 & 4 & 7 & 3 & 0 \\
\hline 1396820 & Orchidaceae & Dactylorhiza elata subsp. sesquipedalis (Willd.) Soó & 8 & 5 & 3 & 9 & 4 & 1 & 0 \\
\hline 1419002 & Orchidaceae & Neotinea commutata (Tod.) R. M. Bateman & 8 & 7 & 5 & 3 & 6 & 3 & 0 \\
\hline 1399002 & Orchidaceae & Himantoglossum adriaticum $\mathrm{H}$. Baumann & 7 & 9 & 4 & 3 & 6 & 2 & 0 \\
\hline 1397015 & Orchidaceae & Serapias strictiflora Welw. ex Da Veiga & 11 & \begin{tabular}{|l|l}
9 & $r$
\end{tabular} & 4 & 3 & 4 & 2 & 0 \\
\hline 1397012 & Orchidaceae & Serapias nurrica Corrias & 11 & 10 & 4 & 2 & 3 & 2 & 0 \\
\hline 1397013 & Orchidaceae & Serapias politisii Renz & 11 & 10 & 4 & 2 & 4 & 2 & 0 \\
\hline 1397014 & Orchidaceae & Serapias bergonii E. G. Camus & 10 & 8 & 5 & 3 & 4 & 2 & 0 \\
\hline 1394035 & Orchidaceae & Ophrys subfusca (Rchb. fil.) Batt. & 8 & 9 & 4 & 3 & 6 & 3 & 0 \\
\hline 1394021 & Orchidaceae & Ophrys mirabilis Geniez \& Melki & 8 & 9 & 4 & 3 & 6 & 3 & 0 \\
\hline 1394023 & Orchidaceae & Ophrys iricolor subsp. maxima (A. Terracc.) Paulus \& Gack & 8 & 9 & 4 & 3 & 6 & 3 & 0 \\
\hline 1394024 & Orchidaceae & Ophrys tardans O. Danesch \& E. Danesch & 8 & 9 & 4 & 3 & 7 & 3 & 0 \\
\hline 1394025 & Orchidaceae & Ophrys argolica H. Fleischm. & 8 & 8 & 5 & 4 & 9 & 3 & 0 \\
\hline 1394026 & Orchidaceae & Ophrys candica (E. Nelson ex Soó) H. Baumann \& Künkele & 10 & 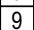 & 5 & 3 & 6 & 3 & 0 \\
\hline 1394027 & Orchidaceae & Ophrys lacaitae Lojac. & 8 & 8 & 4 & 3 & 6 & 3 & 0 \\
\hline 1394028 & Orchidaceae & Ophrys oxyrrhynchos Tod. & 8 & 9 & 4 & 3 & 6 & 3 & 0 \\
\hline 1394017 & Orchidaceae & Ophrys exaltata Ten. & 8 & 9 & 4 & 3 & 6 & 3 & 0 \\
\hline 1394029 & Orchidaceae & Ophrys incubacea Bianca & 8 & 8 & 5 & 4 & 9 & 3 & 0 \\
\hline 1394030 & Orchidaceae & Ophrys splendida Gölz \& H. R. Reinhard & 8 & 8 & 5 & 4 & 9 & 3 & 0 \\
\hline 1394031 & Orchidaceae & Ophrys sipontensis R. Lorenz \& Gembardt & 8 & 9 & 5 & 4 & 7 & 3 & 0 \\
\hline 1394032 & Orchidaceae & Ophrys panattensis Scrugli, Cogoni \& Pessei & 8 & 9 & 5 & 3 & 6 & 3 & 0 \\
\hline 1394033 & Orchidaceae & Ophrys tarentina Gölz \& H. R. Reinhard & 8 & 8 & 5 & 4 & 6 & 3 & 0 \\
\hline 1394037 & Orchidaceae & Ophrys promontorii O. Danesch \& E. Danesch & 8 & 8 & 5 & 4 & 7 & 3 & 0 \\
\hline 1394052 & Orchidaceae & Ophrys araneola Rchb. & 8 & 8 & 4 & 4 & 9 & 3 & 0 \\
\hline 1394051 & Orchidaceae & Ophrys benacensis (Reisigl) O. Danesch, E. Danesch \& Ehrend. & 8 & 8 & 4 & 3 & 6 & 3 & 0 \\
\hline 1394047 & Orchidaceae & Ophrys bertoloniiformis O. \& E. Danesch & 8 & 10 & 3 & 3 & 6 & 3 & 0 \\
\hline 1394053 & Orchidaceae & Ophrys biscutella O. \& E. Danesch & 8 & 8 & 5 & 4 & 9 & 3 & 0 \\
\hline 1394048 & Orchidaceae & Ophrys calliantha Bartolo \& Pulv. & 8 & 9 & 4 & 3 & 6 & 3 & 0 \\
\hline 1394046 & Orchidaceae & Ophrys conradiae F. Melki \& R. Deschatres & 8 & 9 & 5 & 3 & 6 & 3 & 0 \\
\hline 1394050 & Orchidaceae & Ophrys crabronifera Mauri & 8 & 8 & 5 & 4 & 8 & 3 & 0 \\
\hline 1394043 & Orchidaceae & Ophrys dinarica R. Kranicev \& P. Delforge & 8 & 9 & 4 & 3 & 6 & 3 & 0 \\
\hline 1394034 & Orchidaceae & Ophrys discors Bianca & 8 & 9 & 4 & 3 & 6 & 3 & 0 \\
\hline 1394049 & Orchidaceae & Ophrys explanata (Lojac.) P. Delforge & 8 & 9 & 4 & 3 & 7 & 3 & 0 \\
\hline 1394036 & Orchidaceae & Ophrys panormitana (Tod.) Soó & 8 & 8 & 5 & 4 & 7 & 3 & 0 \\
\hline
\end{tabular}


Annex 1. continued.

\begin{tabular}{|c|c|c|c|c|c|c|c|c|c|}
\hline 1394042 & Orchidaceae & Ophrys tetraloniae W. P. Teschner & 8 & 9 & 4 & 3 & 7 & 3 & 0 \\
\hline 1394041 & Orchidaceae & Ophrys tyrrhena Gölz \& H. R. Reinhard & 8 & 9 & 4 & 3 & 6 & 3 & 0 \\
\hline 1482011 & Orchidaceae & Epipactis meridionalis H. Baumann \& R. Lorenz & 3 & 5 & 5 & 5 & 7 & 5 & 0 \\
\hline 1482015 & Orchidaceae & Epipactis ioessa Bongiorni, De Vivo, Fori \& Romolini & 2 & 5 & 4 & 6 & 8 & 6 & 0 \\
\hline 1482016 & Orchidaceae & Epipactis autumnalis Doro & 3 & 5 & 6 & 5 & 7 & 5 & 0 \\
\hline 1482017 & Orchidaceae & Epipactis zaupolensis (Barbaro \& Kreutz) Bongiorni, De Vivo \& Fori & 5 & 6 & 2 & 3 & 8 & 3 & 0 \\
\hline 1482021 & Orchidaceae & Epipactis bugacensis Robatsch & 3 & 5 & 6 & 5 & 7 & 5 & 0 \\
\hline 1482018 & Orchidaceae & Epipactis thesaurensis Agrezzi, Ovatoli \& Bongiorni & 7 & 6 & 2 & 3 & 5 & 3 & 0 \\
\hline 1482012 & Orchidaceae & Epipactis persica (Soó) Nannf. & 4 & 6 & 5 & 4 & $\mathrm{X}$ & 4 & 0 \\
\hline 1482014 & Orchidaceae & Epipactis greuteri $\mathrm{H}$. Baumann \& Künkele & 3 & 5 & 6 & 5 & 7 & 5 & 0 \\
\hline 1482013 & Orchidaceae & Epipactis placentina Bongiorni \& Grünanger & 7 & 6 & 2 & 3 & 8 & 3 & 0 \\
\hline 1482019 & Orchidaceae & Epipactis savelliana Bongiorni, De Vivo \& Fori & 5 & 6 & 2 & 3 & 8 & 3 & 0 \\
\hline 1482020 & Orchidaceae & Epipactis flaminia P. R. Savelli \& Aless. & 3 & 6 & 5 & 5 & 7 & 5 & 0 \\
\hline 1482010 & Orchidaceae & Epipactis aspromontana Bartolo, Pulv. \& Robatsch & 3 & 6 & 5 & 5 & 7 & 5 & 0 \\
\hline 1483002 & Orchidaceae & Limodorum trabutianum Batt. & 4 & 7 & 5 & 4 & 8 & 0 & 0 \\
\hline 1483003 & Orchidaceae & Limodorum brulloi Bartolo \& Pulv. & 4 & 7 & 5 & 4 & 8 & 0 & 0 \\
\hline provv06 & Orchidaceae & Bletilla striata Rchb.fil. & 6 & 7 & 4 & 4 & 5 & 5 & 0 \\
\hline 0530001 & Arecaceae & Trachycarpus fortunei (Hook.) H. Wendl. & 7 & 7 & 6 & 3 & 6 & 3 & 0 \\
\hline 0528003 & Arecaceae & Phoenix dactylifera L. & 11 & 10 & 4 & 5 & $\mathrm{X}$ & 4 & 0 \\
\hline 0528002 & Arecaceae & Phoenix canariensis Chabaud & 11 & 10 & 2 & 4 & $\mathrm{X}$ & 4 & 0 \\
\hline 0049002 & Typhaceae & Typha domingensis (Pers.) Steud. & 8 & 7 & 5 & 10 & 6 & 7 & 0 \\
\hline 0936043 & Juncaceae & Juncus valvatus Link subsp. valvatus & 7 & 7 & 4 & 8 & 6 & 5 & 0 \\
\hline 0936061 & Juncaceae & Juncus minutulus (Albert \& Jahand.) Prain & 4 & 7 & 5 & 6 & 4 & 1 & 0 \\
\hline 0936028 & Juncaceae & Juncus ranarius Songeon \& E. P. Perrier & 7 & 7 & 5 & 9 & 6 & 5 & 1 \\
\hline 0937034 & Juncaceae & Luzula sicula Parl. & 4 & 4 & 4 & 5 & 2 & 3 & 0 \\
\hline 0937035 & Juncaceae & Luzula divulgatiformis Bacic \& Jogan & 7 & 2 & 5 & 7 & 4 & 3 & 0 \\
\hline 0937037 & Juncaceae & Luzula alpina Hoppe & 7 & 2 & 5 & 7 & 4 & 3 & 0 \\
\hline 0937036 & Juncaceae & Luzula expectata Bacic \& Jogan & 7 & 2 & 5 & 7 & 4 & 3 & 0 \\
\hline 0525006 & Cyperaceae & Carex vulpina L. & 7 & 4 & 6 & 7 & 3 & 4 & 0 \\
\hline 0525008 & Cyperaceae & Carex vulpinoidea Michx. & 4 & 4 & 4 & 9 & 7 & 6 & 0 \\
\hline 0525201 & Cyperaceae & Carex randalpina B. Walln. & 7 & 4 & 7 & 9 & 6 & 4 & 0 \\
\hline 0525082 & Cyperaceae & Carex hordeistichos Vill. & 5 & 7 & 5 & 3 & 3 & 4 & 0 \\
\hline 0525198 & Cyperaceae & Carex brevicollis DC. & 5 & 4 & 7 & 4 & $\mathrm{X}$ & 2 & 0 \\
\hline 0525204 & Cyperaceae & Carex clavaeformis Hoppe & 7 & 5 & 5 & 6 & 8 & $\bar{X}$ & 0 \\
\hline 0525203 & Cyperaceae & Carex praetutiana Parl. & 7 & 5 & 5 & 6 & 8 & $\mathrm{X}$ & 0 \\
\hline 0468021 & Cyperaceae & Scirpus georgianus Harp. R. M. & 8 & 4 & 5 & 9 & 5 & 2 & 0 \\
\hline 0468620 & Cyperaceae & Schoenoplectus carinatus (Sm.) Palla & 8 & 7 & 6 & 10 & 8 & 4 & 1 \\
\hline 0468610 & Cyperaceae & Schoenoplectus juncoides (Roxb.) V. I. Krecz. & 8 & 7 & 5 & 11 & 7 & 5 & 0 \\
\hline 0468503 & Cyperaceae & Isolepis pseudosetacea (Daveau) Gand. & 8 & 6 & 4 & 9 & 4 & 1 & 0 \\
\hline 0469019 & Cyperaceae & Eleocharis pellucida J. \& C. Presl & 8 & 6 & 5 & 10 & 3 & 3 & 0 \\
\hline 0471001 & Cyperaceae & Fimbristylis bisumbellata (Forssk.) Bubani & 8 & 6 & 4 & 9 & 4 & 2 & 0 \\
\hline 0471004 & Cyperaceae & Fimbristylis cioniana Savi & 8 & 6 & 4 & 9 & 4 & 2 & 0 \\
\hline 0459031 & Cyperaceae & Cyperus badius Desf. & 8 & 7 & 5 & 11 & 5 & 5 & 0 \\
\hline 0459035 & Cyperaceae & Cyperus dives $\mathrm{L}$. & 8 & 8 & 3 & 9 & 5 & 6 & 0 \\
\hline 0459010 & Cyperaceae & Cyperus involucratus Rottb. & 9 & 9 & 3 & 9 & 5 & 6 & 0 \\
\hline 0459034 & Cyperaceae & Cyperus hamulosus M. Bieb. & 9 & 8 & 4 & 8 & 5 & 5 & 0 \\
\hline 0459030 & Cyperaceae & Cyperus alopecuroides Rottb. & 8 & 8 & 3 & 9 & 5 & 6 & 0 \\
\hline 0459038 & Cyperaceae & Cyperus brevifolioides Thieret \& Delahoussaye & 7 & 7 & 5 & 10 & 5 & 5 & 0 \\
\hline 0204009 & Poaceae & Phalaris hirtiglumis (Trab.) Baldini & 7 & 7 & 5 & 4 & 6 & 4 & 0 \\
\hline 0204902 & Poaceae & Phalaroides rotgesii (Husnot) Holub & 7 & 7 & 5 & 4 & 6 & 4 & 0 \\
\hline 0378057 & Poaceae & Poa perligularis H. Scholz & 8 & 8 & 4 & 2 & 4 & 1 & 0 \\
\hline 0378038 & Poaceae & Poa timoleontis Heldr. ex Boiss. & 8 & 8 & 4 & 2 & 4 & 1 & 0 \\
\hline 0385914 & Poaceae & Vulpia setacea Parl. & 8 & 9 & 4 & 2 & 4 & 2 & 0 \\
\hline 0385601 & Poaceae & Ctenopsis gypsophila (Hackel) Paunero & 10 & 10 & 3 & 1 & 7 & 1 & 0 \\
\hline 0387009 & Poaceae & Catapodium pauciflorum (Merino) Brullo, Giusso, Miniss. \& Spamp. & 11 & 9 & 3 & 1 & $\bar{X}$ & 1 & 2 \\
\hline 0387011 & Poaceae & Catapodium zwierleinii (Lojac.) Brullo & 11 & 10 & 3 & 1 & $\bar{X}$ & 1 & 2 \\
\hline 0385915 & Poaceae & Vulpiella tenuis (Tineo) Kerguélen & 11 & 10 & 3 & 1 & 5 & 1 & 2 \\
\hline 0385192 & Poaceae & Festuca luedii (Markgr.-Dann.) Foggi, Gr. Rossi, Parolo \& Wallossek & 8 & 5 & 5 & 3 & 6 & 2 & 0 \\
\hline 0385187 & Poaceae & Festuca pseudovaria Vetter & 9 & 2 & 5 & 3 & 3 & 2 & 0 \\
\hline 0385193 & Poaceae & Festuca winnebachensis (Wallossek \& Markgr.-Dann.) Foggi, Gr. Rossi, Parolo \& Wallossek & 9 & 2 & 5 & 3 & 3 & 2 & 0 \\
\hline 0385185 & Poaceae & Festuca melanopsis Foggi, Gr. Rossi \& Signorini & 8 & 2 & 5 & 4 & 6 & 4 & 0 \\
\hline 0385191 & Poaceae & Festuca rivularis Boiss. subsp. rivularis & 8 & 4 & 5 & 6 & 4 & 3 & 0 \\
\hline 0385176 & Poaceae & Festuca alfrediana Foggi \& Signorini subsp. alfrediana & 9 & 4 & 5 & 3 & 6 & 2 & 0 \\
\hline 0385177 & Poaceae & Festuca austrodolomitica Pils \& Prosser & 9 & 3 & 5 & 3 & 6 & 2 & 0 \\
\hline
\end{tabular}


Annex 1. continued.

\begin{tabular}{|c|c|c|c|c|c|c|c|c|c|}
\hline 0385195 & Poaceae & Festuca plonkae Foggi \& Signorini & 9 & 2 & 4 & 2 & 3 & 2 & 0 \\
\hline 0385181 & Poaceae & Festuca humifusa Brullo \& Guarino & 11 & 10 & 5 & 1 & 6 & 2 & 0 \\
\hline 0385194 & Poaceae & Festuca marginata subsp. gallica (Hack. ex Charrel) Breistr. & 11 & 6 & 5 & 1 & 6 & 2 & 0 \\
\hline 0385113 & Poaceae & Festuca ovina L. & 8 & 8 & 6 & 3 & 8 & 3 & 0 \\
\hline 0385190 & Poaceae & Festuca veneris Gr. Rossi, Foggi \& Signorini & 11 & 8 & 5 & 2 & 6 & 2 & 0 \\
\hline 0385188 & Poaceae & Festuca riccerii Foggi \& Gr. Rossi & 8 & 3 & 4 & 3 & 4 & 2 & 0 \\
\hline 0385182 & Poaceae & Festuca imperatrix Catonica & 9 & 2 & 5 & 3 & 6 & 2 & 0 \\
\hline 0385180 & Poaceae & Festuca gamisansii subsp. aethaliae Signorini \& Foggi & 9 & 4 & 3 & 3 & 6 & 2 & 0 \\
\hline 0385183 & Poaceae & Festuca laevigata Gaudin & 9 & 2 & 5 & 3 & 6 & 2 & 0 \\
\hline 0385178 & Poaceae & Festuca billyi Kerguélen \& Plonka & 8 & 3 & 4 & 3 & 4 & 2 & 0 \\
\hline 0385097 & Poaceae & Festuca arvernensis subsp. costei (St.-Yves) Auquier \& Kerguélen & 9 & 3 & 5 & 3 & 6 & 2 & 0 \\
\hline 0385208 & Poaceae & Festuca bauzanina (Pils) Arndt & 8 & 6 & 8 & 3 & 6 & 2 & 0 \\
\hline 0385160 & Poaceae & Festuca stricta Host & 8 & 4 & 5 & 3 & 6 & 2 & 0 \\
\hline 0385198 & Poaceae & Schedonorus uechtritzianus (Wiesb.) Holub. & 9 & 8 & 5 & 6 & 8 & 6 & 0 \\
\hline 0395008 & Poaceae & Lolium siculum Parl. & 8 & 8 & 5 & 4 & $\mathrm{X}$ & 6 & 0 \\
\hline 0387010 & Poaceae & Desmazeria pignattii Brullo \& Pavone & 11 & 10 & 3 & 1 & $\mathrm{X}$ & 1 & 2 \\
\hline 0324002 & Poaceae & Sesleriella leucocephala (DC.) Deyl & 9 & 2 & 4 & 3 & 8 & 2 & 0 \\
\hline 0324032 & Poaceae & Sesleria pichiana Foggi, Gr. Rossi \& Pignotti & 5 & 6 & 5 & 4 & 8 & 2 & 0 \\
\hline 0324033 & Poaceae & Sesleria apennina Ujhelyi & 10 & 4 & 4 & 2 & 7 & 4 & 0 \\
\hline 0324034 & Poaceae & Sesleria calabrica (Deyl) Di Pietro & 10 & 4 & 4 & 2 & 7 & 4 & 0 \\
\hline 0402005 & Poaceae & Parapholis pycnantha (Hack.) C. E. Hubb. & 11 & 9 & 4 & 5 & 7 & 2 & 7 \\
\hline 0402002 & Poaceae & Parapholis marginata Runemark & 11 & 9 & 4 & 5 & 7 & 2 & 7 \\
\hline 0402003 & Poaceae & Parapholis filiformis (Roth) C. E. Hubb. & 11 & 9 & 4 & 5 & 7 & 2 & 7 \\
\hline 0346014 & Poaceae & Koeleria australis Kerner & 11 & 7 & 6 & 3 & 7 & 1 & 0 \\
\hline 0346015 & Poaceae & Koeleria callieri (Domin) Ujhelyi & 11 & 7 & 6 & 3 & 7 & 1 & 0 \\
\hline 0346016 & Poaceae & Koeleria insubrica Brullo, Giusso \& Minissale & 11 & 7 & 6 & 3 & 7 & 1 & 0 \\
\hline 0346017 & Poaceae & Koeleria lucana Brullo, Giusso \& Minissale & 11 & 7 & 6 & 3 & 7 & 1 & 0 \\
\hline 0271926 & Poaceae & Trisetaria burnoufii (Req. ex Parl.) Banfi \& Soldano & 8 & 9 & 5 & 8 & 8 & 6 & 0 \\
\hline 0245004 & Poaceae & Gastridium phleoides (Nees \& Meyen) C. E. Hubb. & 8 & 9 & 4 & 2 & 4 & 2 & 0 \\
\hline 0273129 & Poaceae & Avenula adsurgens subsp. ausserdorferi (Asch. \& Graebn.) Sauer \& Chmelitschek & 8 & 3 & 5 & 7 & 4 & 4 & 0 \\
\hline 0273214 & Poaceae & Helictotrichon petzense Melzer & 8 & 4 & 6 & 5 & 8 & 3 & 0 \\
\hline 0275004 & Poaceae & Arrhenatherum sardoum (Em. Schmid) Brullo, Minissale \& Spampinato & 8 & 5 & 4 & 5 & 7 & 7 & 0 \\
\hline 0275005 & Poaceae & Arrhenatherum bulbosum (Willd.) C. Presl & 8 & 5 & 5 & 5 & 7 & 7 & 0 \\
\hline 0275006 & Poaceae & Arrhenatherum nebrodense Brullo, Minissale \& Spampinato & 8 & 5 & 5 & 5 & 7 & 7 & 0 \\
\hline 0273018 & Poaceae & Avena lusitanica (Tab. Morais) Baum & 8 & 8 & 4 & 3 & 7 & 2 & 0 \\
\hline 0273017 & Poaceae & Avena matritensis B. R. Baum & 8 & 8 & 5 & 3 & 7 & 2 & 0 \\
\hline 0273005 & Poaceae & Avena saxatilis (Lojac.) Rocha Afonso & 8 & 8 & 5 & 3 & 7 & 2 & 0 \\
\hline 0273016 & Poaceae & Avena wiestii Steud. & 8 & 8 & 5 & 3 & 7 & 2 & 0 \\
\hline 0273003 & Poaceae & Avena longiglumis Dur. & 6 & 7 & 6 & 6 & 7 & 2 & 0 \\
\hline 0273015 & Poaceae & Avena insularis Ladiz. & 9 & 8 & 5 & 3 & 7 & 2 & 0 \\
\hline 0273012 & Poaceae & Avena byzantina K. Koch & 8 & 8 & 5 & 3 & 7 & 2 & 0 \\
\hline 0273007 & Poaceae & Avena strigosa Schreb. & 8 & 7 & 6 & 5 & 6 & 6 & 0 \\
\hline 0273001 & Poaceae & Avena clauda Dur. & 6 & 7 & 6 & 6 & 7 & 2 & 0 \\
\hline 0242013 & Poaceae & Agrostis tenerrima Trin. & 8 & 9 & 4 & 2 & 3 & 1 & 0 \\
\hline 0242026 & Poaceae & Agrostis monteluccii (Selvi) Banfi & 11 & 8 & 4 & 2 & 6 & 2 & 0 \\
\hline 0247015 & Poaceae & Calamagrostis corsica (Hack.) D. Prain & 7 & 3 & 4 & 5 & 8 & 3 & 0 \\
\hline 0331003 & Poaceae & Arundo collina Ten. & 10 & 9 & 4 & 5 & 4 & 4 & 1 \\
\hline 0383009 & Poaceae & Glyceria spicata Guss. & 7 & 6 & 5 & 9 & 5 & 5 & 0 \\
\hline 0389037 & Poaceae & Ceratochloa carinata (Hook. \& Arnott) Tutin & 8 & 8 & 5 & 2 & 5 & 3 & 0 \\
\hline 0393011 & Poaceae & Brachypodium caespitosum (Host) Roem. \& Schult. & 8 & 6 & 4 & 5 & 8 & 4 & 0 \\
\hline 0393010 & Poaceae & Brachypodium genuense (DC.) Roem. \& Schult. & 8 & 7 & 6 & 4 & 7 & 3 & 0 \\
\hline 0411902 & Poaceae & Taeniatherum asperum (Simonk.) Nevski & 6 & 6 & 4 & 4 & 5 & 4 & 0 \\
\hline 0410111 & Poaceae & Psathyrostachys juncea (Fisch.) Nevski & 8 & 8 & 5 & 5 & 5 & 5 & 0 \\
\hline 0410002 & Poaceae & Hordeum distichon $\mathrm{L}$. & 8 & 8 & 5 & 5 & 5 & 5 & 0 \\
\hline 0405031 & Poaceae & Agropyron desertorum (Fisch. ex Link) Schult. & 11 & 7 & 5 & 5 & 7 & 7 & 3 \\
\hline 0405032 & Poaceae & Elytrigia scirpea (C. Presl) Holub & 10 & 6 & 4 & 5 & 6 & 6 & 2 \\
\hline 0405033 & Poaceae & Elytrigia obtusiflora (DC.) Tzvelev & 10 & 6 & 4 & 5 & 6 & 6 & 2 \\
\hline 0405034 & Poaceae & Elytrigia sartorii (Boiss. \& Heldr.) H. Scholz & 10 & 6 & 4 & 5 & 6 & 6 & 2 \\
\hline 0405036 & Poaceae & Elytrigia acuta (DC.) Kerguélen ex Carreras Martínez & 11 & 7 & 5 & 5 & 6 & 7 & 3 \\
\hline 0408009 & Poaceae & Triticum compactum Host & 8 & 8 & 5 & 5 & 5 & 5 & 0 \\
\hline 0408007 & Poaceae & Triticum spelta $\mathrm{L}$. & 8 & 8 & 5 & 5 & 5 & 5 & 0 \\
\hline 0408003 & Poaceae & Triticum dicoccon Schrank & 8 & 8 & 5 & 5 & 5 & 5 & 0 \\
\hline 0408006 & Poaceae & Triticum polonicum L. & 8 & 8 & 5 & 5 & 5 & 5 & 0 \\
\hline 0408912 & Poaceae & Triticum biunciale (Vis.) K. Richter & 8 & 8 & 5 & 5 & 5 & 5 & 0 \\
\hline
\end{tabular}


Annex 1. continued.

\begin{tabular}{|c|c|c|c|c|c|c|c|c|c|}
\hline 0408901 & Poaceae & Triticum speltoides (Tausch) Gren. ex K. Richter & 8 & 8 & 5 & 5 & 5 & 5 & 0 \\
\hline 0209048 & Poaceae & Stipa eriocaulis Borbás & 8 & 8 & 8 & 1 & 8 & 2 & 0 \\
\hline 0209049 & Poaceae & Stipa etrusca Moraldo & 8 & 8 & 8 & 1 & 8 & 2 & 0 \\
\hline 0209053 & Poaceae & Stipa veneta Moraldo & 9 & 7 & 7 & 1 & 8 & 1 & 0 \\
\hline 0209047 & Poaceae & Stipa dasyvaginata subsp. apenninicola Martinovský \& Moraldo & 8 & 8 & 8 & 1 & 8 & 2 & 0 \\
\hline 0209052 & Poaceae & Stipa oligotricha Moraldo & 9 & 9 & 7 & 2 & 8 & 2 & 0 \\
\hline 0209007 & Poaceae & Stipa epilosa Martinovský & 8 & 8 & 8 & 1 & 8 & 2 & 0 \\
\hline 0209046 & Poaceae & Stipa aquilana Moraldo & 8 & 8 & 8 & 1 & 8 & 2 & 0 \\
\hline 0209050 & Poaceae & Stipa gussonei Moraldo & 10 & 9 & 7 & 2 & 8 & 2 & 0 \\
\hline 0209801 & Poaceae & Amelichloa caudata (Trin.) Arriaga \& Barkworth & 9 & 10 & 3 & 1 & 1 & 5 & 0 \\
\hline 0209054 & Poaceae & Nassella formicarum (Delile) Barkworth & 9 & 8 & 5 & 2 & 8 & 2 & 0 \\
\hline 0209055 & Poaceae & Nassella hyalina (Nees) Barkworth & 9 & 8 & 5 & 2 & 8 & 2 & 0 \\
\hline 0210005 & Poaceae & Piptatherum holciforme (M. Bieb.) Roem. \& Schultes & 7 & 7 & 4 & 4 & 7 & 5 & 0 \\
\hline 0210003 & Poaceae & Piptatherum paradoxum (L.) P. Beauv. & 7 & 7 & 4 & 4 & 7 & 5 & 0 \\
\hline 0376002 & Poaceae & Schismus arabicus Nees & 10 & 11 & 5 & 3 & 7 & 4 & 0 \\
\hline 0329001 & Poaceae & Cortaderia selloana (Schult.) Asch. \& Graebn. & 8 & 9 & 5 & 6 & 5 & 6 & 0 \\
\hline 0341011 & Poaceae & Eragrostis mexicana subsp. virescens (J. Presl) S. D. Koch \& Sánchez Vega & 8 & 8 & 5 & 4 & 6 & 3 & 0 \\
\hline 0341010 & Poaceae & Eragrostis frankii C. A. Mey. ex Steud. & 8 & 8 & 5 & 4 & 6 & 3 & 0 \\
\hline 0341015 & Poaceae & Eragrostis capillaris Nees & 8 & 8 & 5 & 4 & 6 & 3 & 0 \\
\hline 0341014 & Poaceae & Eragrostis curvula (Schrad.) Nees & 8 & 8 & 5 & 4 & $\mathrm{X}$ & 3 & 0 \\
\hline 0337002 & Poaceae & Diplachne fusca (L.) P. Beauv. ex Roem. \& Schultes & 9 & 8 & 3 & 6 & 8 & 6 & 0 \\
\hline 0337003 & Poaceae & Diplachne fascicularis (Lam.) P. Beauv. & 9 & 8 & 3 & 6 & 8 & 6 & 0 \\
\hline 0337004 & Poaceae & Diplachne uninervia (J. Presl) Parodi & 9 & 8 & 3 & 6 & 8 & 6 & 0 \\
\hline 0304003 & Poaceae & Eleusine tristachya (Lam.) Lam. & 10 & 8 & 4 & 2 & 7 & 2 & 0 \\
\hline 0304002 & Poaceae & Eleusine coracana (L.) Asch. \& Gr. & 10 & 8 & 5 & 2 & 7 & 2 & 0 \\
\hline 0150001 & Poaceae & Zoysia matrella (L.) Merr. & 8 & 8 & 6 & 4 & $X$ & 4 & 1 \\
\hline 0208002 & Poaceae & Aristida longispica Poir. & 9 & 11 & 5 & 1 & 7 & 1 & 0 \\
\hline 0193001 & Poaceae & Oryza sativa L. & 7 & 8 & 6 & 9 & 8 & 8 & 0 \\
\hline 0166008 & Poaceae & Panicum philadelphicum Bernh. ex Trin. & 6 & 8 & 5 & 4 & 5 & 5 & 0 \\
\hline 0166601 & Poaceae & Dichanthelium acuminatum subsp. implicatum (Scribn. ex Nash) Freckman \& Lelong & 8 & 8 & 5 & 4 & $\bar{X}$ & 4 & 0 \\
\hline 0166602 & Poaceae & Dichanthelium clandestinum (L.) Gould & 8 & 8 & 5 & 4 & $\mathrm{X}$ & 4 & 0 \\
\hline 0166909 & Poaceae & Echinochloa microstachya (Wiegand) Rydb. & 8 & 8 & 5 & 9 & 6 & 8 & 0 \\
\hline 0166805 & Poaceae & Digitaria violascens Link & 7 & 7 & 5 & 3 & 6 & 4 & 0 \\
\hline 0161007 & Poaceae & Paspalum vaginatum $\mathrm{Sw}$. & 11 & 9 & 5 & 3 & 6 & 8 & 0 \\
\hline 0161008 & Poaceae & Paspalum exaltatum J. Presl \& C. Presl & 10 & 8 & 5 & 4 & 6 & 8 & 0 \\
\hline 0171008 & Poaceae & Setaria faberi F. Herm. & 7 & 6 & 5 & 4 & 6 & 7 & 0 \\
\hline 0171009 & Poaceae & Setaria pycnocoma (Steudel) Henr. ex Nakai & 7 & 7 & 5 & 4 & 5 & 6 & 0 \\
\hline 0171007 & Poaceae & Setaria adhaerens (Forssk.) Chiov. & 7 & 7 & 5 & 4 & 7 & 8 & 0 \\
\hline 0171004 & Poaceae & Setaria italica (L.) P. Beauv. & 7 & 8 & 5 & 4 & 6 & 8 & 0 \\
\hline 0174003 & Poaceae & Cenchrus longispinus (Hack.) Fernald & 9 & 9 & 0 & 1 & $\bar{X}$ & 1 & 0 \\
\hline 0110001 & Poaceae & Miscanthus sinensis Anderss. & 10 & 12 & 6 & 1 & $\mathrm{X}$ & 1 & 0 \\
\hline 0111001 & Poaceae & Saccharum spontaneum subsp. aegyptiacum (Willd.) Hack. & 11 & 11 & 4 & 4 & 5 & 4 & 0 \\
\hline 0111002 & Poaceae & Saccharum officinarum $L$. & 12 & 11 & 4 & 5 & 5 & 6 & 0 \\
\hline 0134903 & Poaceae & Sorghum bicolor (L.) Moench & 8 & 9 & 4 & 6 & 6 & 8 & 0 \\
\hline 0134004 & Poaceae & Dichanthium annulatum (Forssk.) Stapf & 11 & 10 & 5 & 2 & 6 & 3 & 0 \\
\hline 0134703 & Poaceae & Bothriochloa laguroides (DC.) Herter & 9 & 7 & 4 & 4 & 4 & 4 & 0 \\
\hline 0134602 & Poaceae & Hyparrhenia sinaica (Delile) Llauradó ex G. López & 11 & 12 & 5 & 2 & 7 & 3 & 0 \\
\hline 0424001 & Poaceae & Pleioblastus pygmaeus (Miq.) Nakai & 7 & 8 & 4 & 3 & 5 & 4 & 0 \\
\hline 0424002 & Poaceae & Pleioblastus chino (Franch. \& Savat.) Makino & 8 & 8 & 4 & 3 & 5 & 4 & 0 \\
\hline 0424003 & Poaceae & Bambusa multiplex (Lour.) Raeuschel ex J. A. Schultes \& J. H. Schultes & 8 & 9 & 5 & 5 & 4 & 6 & 0 \\
\hline 0414003 & Poaceae & Pseudosasa japonica (Sieb. \& Zucc. ex Steudel) Makino ex Nakai & 8 & 8 & 5 & 3 & 5 & 6 & 0 \\
\hline 0417001 & Poaceae & Phyllostachys nigra (Lodd.) Munro & 8 & 7 & 5 & 3 & 5 & 6 & 0 \\
\hline 0417002 & Poaceae & Phyllostachys edulis (Carrière) Houz. & 8 & 8 & 5 & 3 & 5 & 6 & 0 \\
\hline 0417003 & Poaceae & Phyllostachys reticulata (Rupr.) K. Koch & 8 & 8 & 5 & 3 & 5 & 6 & 0 \\
\hline 0417004 & Poaceae & Phyllostachys aurea (Carrière) A. Rivière \& C. Rivière & 8 & 8 & 5 & 3 & 5 & 6 & 0 \\
\hline 0417005 & Poaceae & Phyllostachys sulphurea (Carrière) Rivière \& C. Rivière & 7 & 8 & 4 & 3 & 5 & 6 & 0 \\
\hline 0417006 & Poaceae & Phyllostachys viridiglaucescens (Carrière) A. Rivière \& C. Rivière & 8 & 7 & 5 & 3 & 5 & 6 & 0 \\
\hline 0918001 & Commelinaceae & Zebrina pendula Schnizl. & 7 & 6 & 5 & 8 & 6 & 2 & 0 \\
\hline 0911003 & Commelinaceae & Tradescantia albiflora Kunth & 7 & 6 & 5 & 8 & 6 & 2 & 0 \\
\hline 0924002 & Pontederiaceae & Heteranthera limosa (Sw.) Willd. & 8 & 8 & 5 & 10 & 6 & 6 & 0 \\
\hline 0924003 & \begin{tabular}{|l|} 
Pontederiaceae \\
\end{tabular} & Heteranthera rotundifolia (Kunth) Griseb. & 8 & 8 & 5 & 10 & 6 & 6 & 0 \\
\hline 0920001 & \begin{tabular}{|l|} 
Pontederiaceae \\
\end{tabular} & Monochoria korsakowii Regel \& Maack & 8 & 7 & 5 & 12 & 7 & 7 & 0 \\
\hline 1363002 & Cannaceae & Canna glauca $\mathrm{L}$. & 9 & 10 & 5 & 5 & 7 & 6 & 0 \\
\hline 1363001 & Cannaceae & Canna indica L. & 9 & 9 & 5 & 5 & 7 & 6 & 0 \\
\hline
\end{tabular}


